\title{
DATA ANALYTICS WITH MLV-VIEWER
}

\author{
Carlos Manuel Oliveira Alves ${ }^{1}$, Manuel Pérez Cota ${ }^{2, *}$ and Miguel Ramón González Castro ${ }^{2, *}$ \\ ${ }^{I}$ Instituto Politécnico de Castelo Branco, Av. D. Pedro Álvares Cabral 12, 6000-Castelo Branco, Portugal \\ ${ }^{2}$ Universidade de Vigo, Rúa Torrecedeira 86; 36208 -Vigo, Spain \\ * Dr.
}

\begin{abstract}
Information visualization is defined as interactive visual representations supported by the computer, in order to increase cognition. The tools and methods applied can help to accelerate the understanding of a large volume of data, improving understanding, especially with multidimensional data sets. Visual analysis methods allow decision makers to combine flexibility, creativity and human knowledge with the enormous storage and processing resources of today's computers to gain insight into complex problems. If we add data mining to advanced visual interfaces, decision makers will be able to interact directly with data analysis, adapting to their tastes and needs. The MLV-Viewer is a decision support system, and its data analytics aspect will be described in this article.
\end{abstract}

\section{KEYWORDS}

Data Analytics in DSS, DSS, Big Data Visualization

\section{INTRODUCTION}

In the late 1960s, a new type of model-oriented information system emerged, Decision Support Systems (DSS) or also called Management Decision Systems (MDS) (Müller et al., 2016). Peter Keen and Charles Stabell, the pioneers of the DSS concept, point out that the concept has evolved from theoretical studies of organizational decision-making at the Carnegie Institute of Technology. However, the origin of the DSS goes back to the beginning of the 20th century, when the military objective was dominant. One of the oldest DSSs was the SAGE (semi-automatic terrestrial environment), designed to unify different images throughout the area and used in the Cold War. The SAGE was the largest computer ever built. According to another approach, the first DSS may have its origins in LEO I (Lyons Electronic Office) in 1951.

Data visualization is a dynamic way to react quickly to new developments using either virtual environments or network technology or new computer graphics algorithms. The user, that is, the decision maker, initially gets a total idea about a scenario before focusing on the details, but the observation in a graphical model of the data provides part of the information. It can also be said that visualization does not just mean seeing, but allows the user to see what is not seen naturally. Visualization is nothing more than a graphical representation of data with the main objective of providing the user with a qualitative and easy understanding of the content of information (which can be data, relationships, perceptions or processes) (Kumar \& Belwal, 2018), transforming objects, numbers and concepts into a form that can be easily interpreted by the human eye. From a set of data, (Katrina E. Barkwell, 2018) says that the essential role of visualization is nothing more than visualizing data to tell stories, selecting, filtering and transforming that data and choosing the correct visualization type (such as bars, line or bubble charts).

The visualization techniques aim to simplify the data and that the representation of models provides relevant information from that same data, facilitating the production of ideas in a short time, especially if we have a large volume of data. That is why the integration of visualization techniques in dynamic and complex systems is highly recommended for data abstraction, allowing the user to quickly navigate through the wide range of data. However, data visualization requires interaction between the user and the system (Ellouzi, Ltifi, \& Ayed, 2016).

The visualization of data in the DSS, despite increasing the requirements for modeling and use, also allows the extraction of relevant information and the interaction with them (Grignard, Drogoul, \& Zucker, 2013). In this way, visual information combined with interaction techniques allows the user to obtain knowledge through visual representations, viewing, searching and analyzing a large volume of data quickly and clearly. 
According to (Cota, Manuel Pérez; Castro \& Domínguez, 2014), visual analysis is important for analysts and decision makers, reducing research by synthesizing large volumes of data as well as facilitating the task of exploration. It is also said that pattern recognition and inference of relationships between data is facilitated through visualizations using graphical interfaces, thereby amplifying cognitive factors and exposing the relationships between the various entities extracted from the data, producing useful information and minimizing the difficulty analysis. Visual analysis systems need to have the ability to identify patterns hidden in the data so that the decision maker can build knowledge from a large volume of data (Yan et al., 2012). Combining data mining algorithms with data visualization, we can reduce the information obtained but make the patterns more visible (Jorgensen et al., 2018), that is, the extraction of information about large volumes of data must use computational data mining methods.

To support knowledge discovery and decision making from large-scale data sets (Yan et al., 2012), new visual analysis systems need to be able to identify hidden patterns in the data which can be critical for further analysis.

In order to extract useful information from large volumes of data, computational data mining methods have the advantage over visualization, as they quickly capture data patterns (Jorgensen et al., 2018). By combining data mining algorithms with visualization, the information obtained can be reduced to make the patterns more visible.

Data mining is a key concept in Big Data Analytics consisting of the application of data science techniques to analyze and explore large volumes of data in order to find useful and significant patterns in that data as a way to facilitate the task of the decision maker, offering dashboards, graphs, or reports (Huru, Leordeanu, Apostol, \& Cristea, 2018). For that, mathematical and statistical methods must be used to understand the data, simulate scenarios, validate hypotheses and make predictions, mainly to perform four types of analysis:

- descriptive analysis: transforms data into meaningful information for the purposes of interpretation, reporting, monitoring and visualization through statistical graphic tools, such as pie charts, bar charts and panels;

- $\quad$ predictive analysis: it is usually defined as an extrapolation of data based on the available data to ensure better decision making;

- $\quad$ prescriptive analysis: it is associated with descriptive and predictive analysis, offering options on how to obtain future benefits or mitigate a future risk, detailing the implications of each decision option;

- exploratory analysis: view unexpected or hidden relationships between data.

This prototype aims to build a universal DSS (MLV-Viewer, meaning Multi Level Visualization Viewer), multi-platform, free of cost and adaptable to the decision maker needs. In this article, the topic will be presented in the "I-Introduction" section, followed by a literature review on the topic "data mining" in the "II-Literature Review" section, in the section "III- New Approach: MLV-Viewer" the prototype will be described and, finally, in the section" IV-Conclusion and Future Work ", some conclusions and future works.

\section{LITERATURE REVIEW}

According to (Ellouzi et al., 2016) (Grignard et al., 2013) (Cota, Manuel Pérez; Castro \& Domínguez, 2014) (Yan et al., 2012) (Jorgensen et al., 2018), DSS provide support for semi-structured and unstructured decision problems, usually interactive computer-based systems to help decision makers use various tools (such as communication, data, document, knowledge technologies and modeling) to assist in decision making in several areas, and the main components are: decision maker; data base; models and procedures; Software.

Decision makers demand better identification of metrics applied to data in order to be able to interpret these complex patterns more easily. For this reason, the area of data mining has been heavily invested, but there is still a gap between efforts to visualize these patterns and complex data recently extracted (Ashraf \& Khan, 2015).

In the work carried out by (Groumpos, 2016), four visual analysis tools were developed to control which layers on a map the user displays to understand the impacts on black ice, these tools are: visual analysis tool, interpolation tool, animation tool and Analysis tool Decision Support.

The study presented by (Alves \& Cota, 2018) states that the use of dynamic symbols (such as size, color, shape or outlines), instead of static symbols, according to the information they represent conveys additional semantics. AgMine (Imen, Chang, Yang and Golchubian, 2016) has an architecture focused on: pre-processing 
of data sets; visualization of data in general lines; visualization of data using spatial interpolation; data mining; analysis and interpolation of scenarios. InVEST (Reschka, Bagschik, Ulbrich, Nolte and Maurer, 2015) exports and adapts the data to create various interactive and abstract data visualizations, using web programming languages. The study (Wu et al., 2008) presents the top 10 algorithms for data mining for both forecasting and interpolation, namely the average, the weighted average, the linear regression, the expected maximum and the multiple imputation.

\section{DATA ANALYTICS WITH MLV-VIEWER}

The prototype developed, MLV-Viewer, and presented below, allows the user to dynamically and interactively select the data he/she wants to view and how to view it.

The developed prototype works in a web environment with a MySQL server as a database system (or any other database management system as long as it uses the SQL language), uses PHP and JavaScript as programming languages, uses canvasJs, Chart.js, JpGraph, HIGHCHARTS and three.js as graphic libraries (see Figure 1).

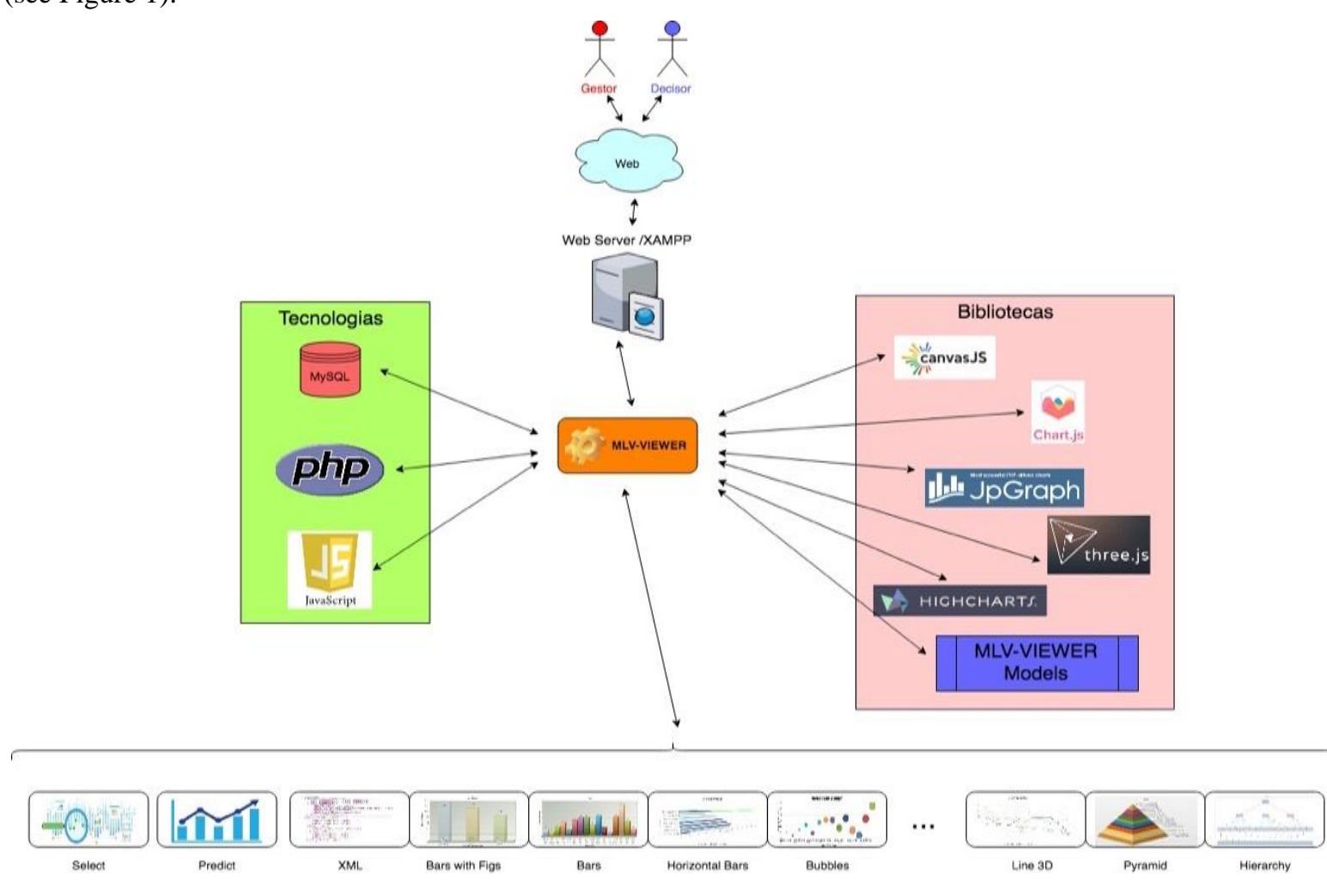

Figure 1. MLV-Viewer Architecture

If the data has not been previously imported into the MySQL database, then he can import both the logical structure (contained in an XML schema file) and the data (contained in an XML file). The MLV-Viewer also allows the export of data to XML and XSD. The user will be able to visualize these data in $1 \mathrm{D} 2 \mathrm{D}, 2 \mathrm{D}+1,3 \mathrm{D}$ and $3 \mathrm{D}+1,3 \mathrm{D}+2,3 \mathrm{D}+3,3 \mathrm{D}+4$, as well as to analyze the data, and these are presented in $2 \mathrm{D}$ or $3 \mathrm{D}+1$ format (see Figure 2). 


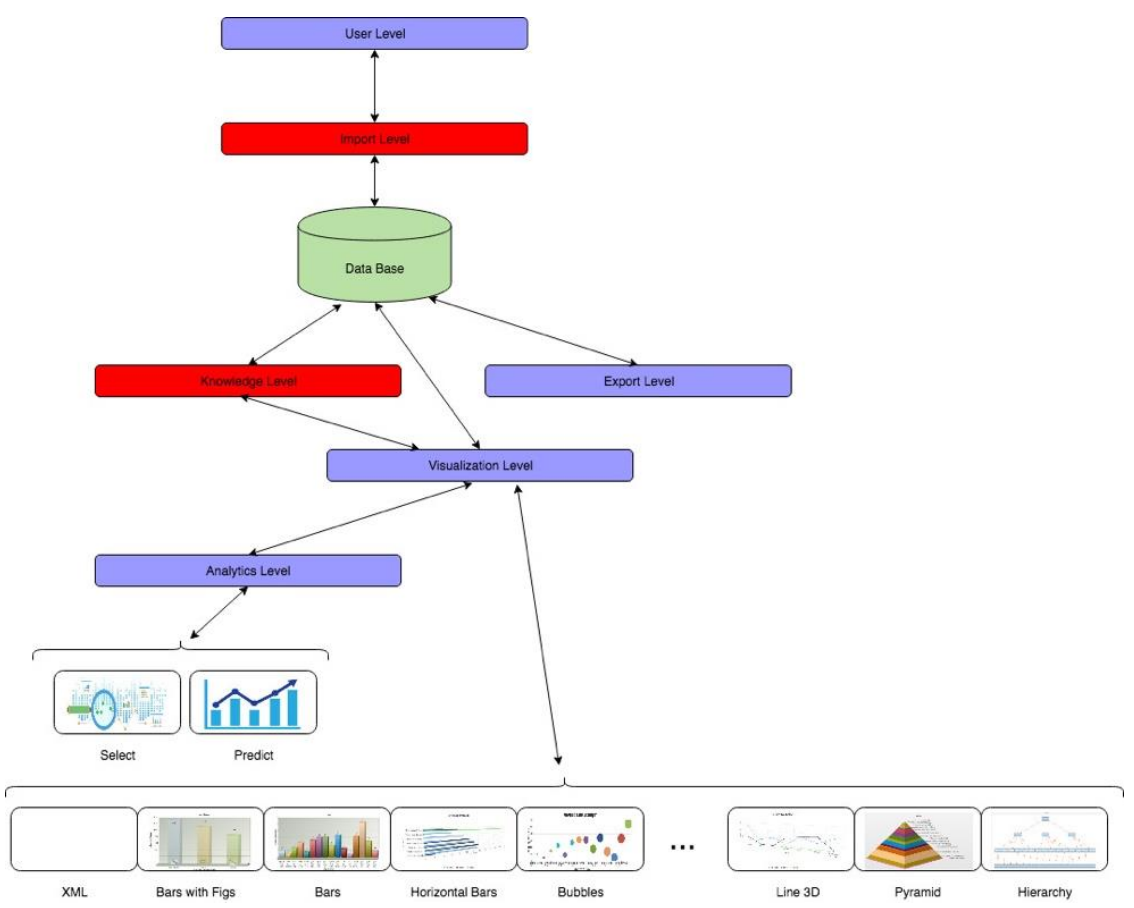

Figure 2. MLV-Viewer Ontology

The user has at his disposal a set of "smart" visualizations (due to the pairing between products / data) and also self-descriptive (when presenting the image associated with that data / product), see Figure 3.

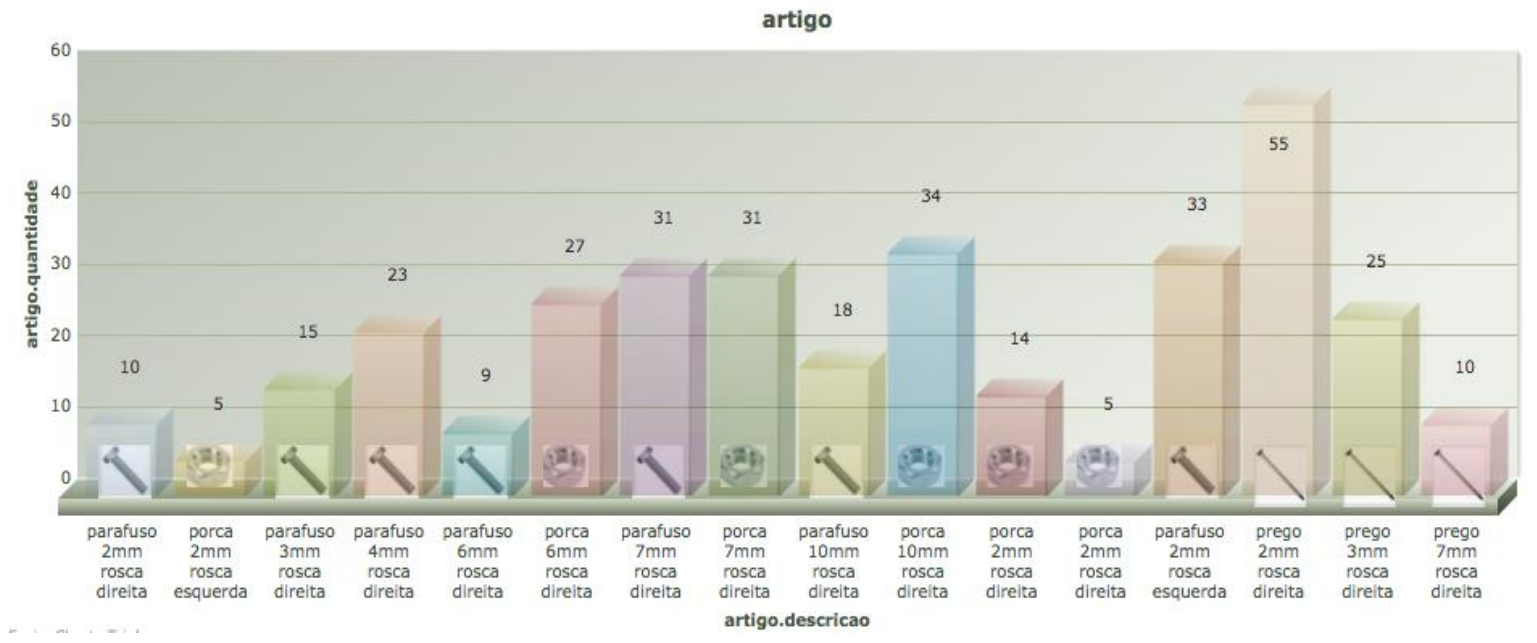

Figure 3. 2D image with bars

To describe the data mining of the MLV-Viewer, the data referring to the products "screw", "nut" and "nail" were chosen, this choice is due to the fact that they are products easily understood by all, but at the same time they can be as complex as you want.

In Figure 4, a 2D visualization, the products are presented in the form of stacked bars, and in this way it is easily noticeable to compare the existence in stock of the various products. 

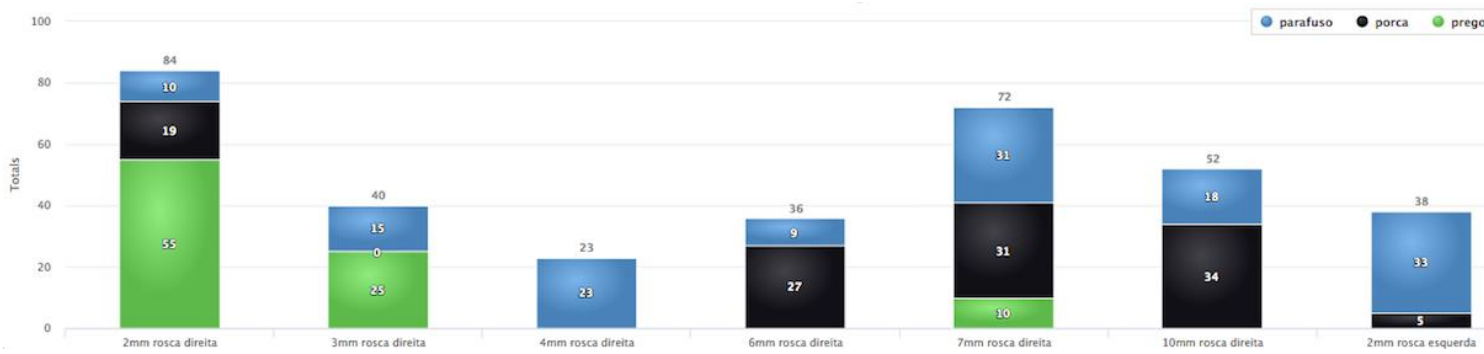

Figure 4. 2D data analysis with stacked bars

In Figure 5, it can be seen quickly and easily that for the products "screw", "nut" and "nail" there is a discrepancy in quantity in stock for the different measures (" $2 \mathrm{~mm} "$, " $3 \mathrm{~mm}$ ", " $4 \mathrm{~mm} "$, ,..) displayed on the bars or through the lines that represent the minimum, average and maximum quantities for each of the different measures. It is also possible to view the total quantity for each of the products in the "cheese".

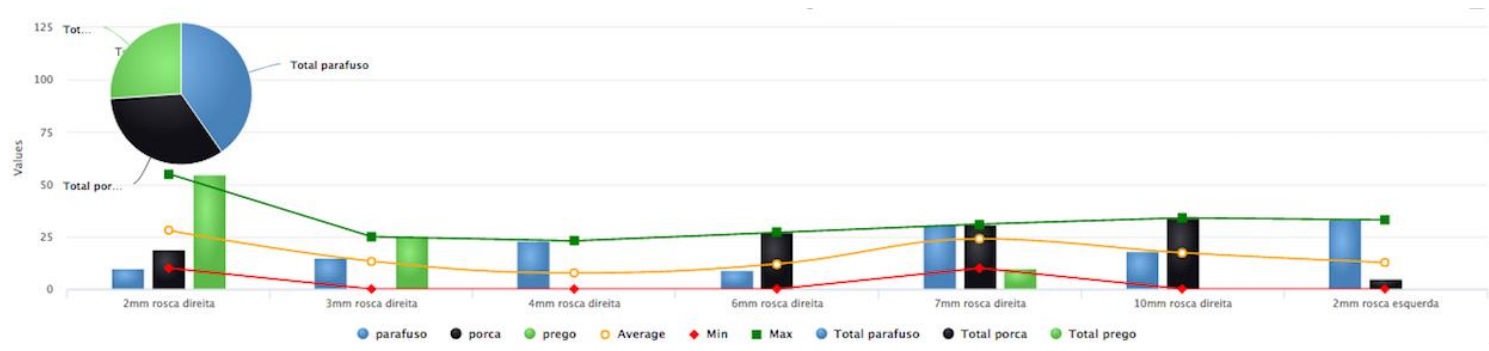

Figure 5. 2D data analysis with bars

Figure 6 is similar to Figure 5, but the bars have been replaced by dots, keeping all other information.

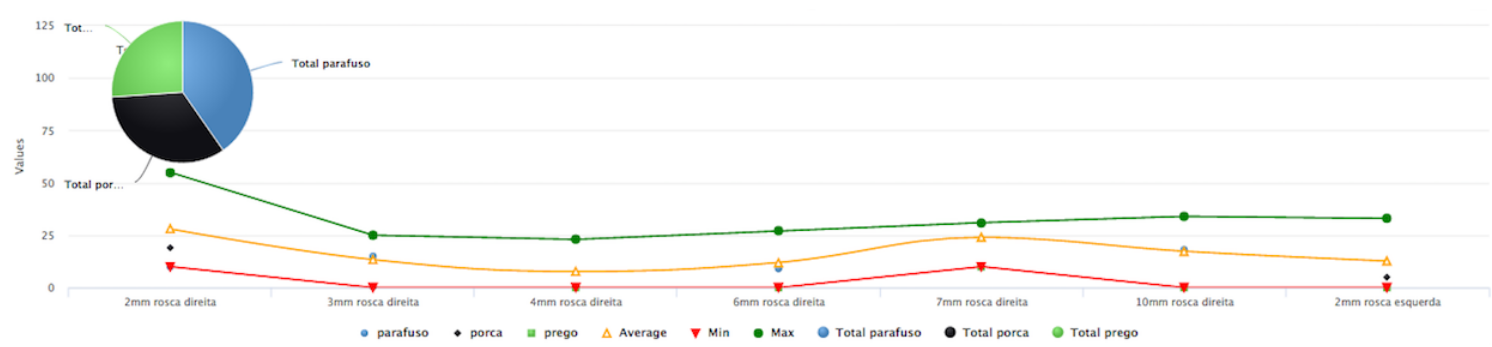

Figure 6. 2D data analysis with points

In Figure 7, a 2D + 1 visualization is achieved by varying the size of the stitch according to the quantity of the respective product.

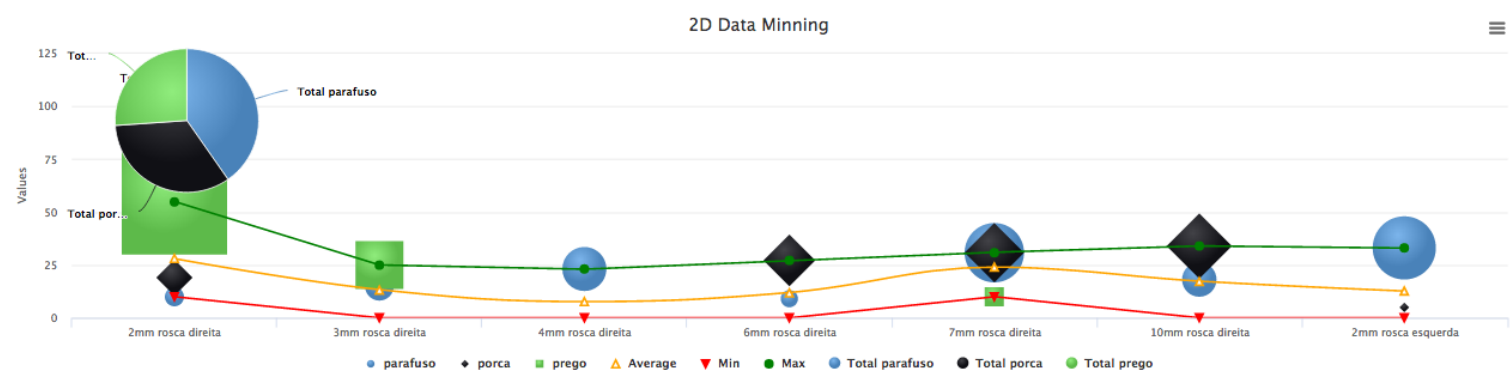

Figure 7. 2D+1 data analysis with radius points

A $3 \mathrm{D}$ view in which the user can rotate and zoom the view is very important when you want to do data mining, and if it is complemented with $+1 \mathrm{D}$ even more. In the following figure (Figure 8), the user can 
dynamically choose what to display on each axis (numeric or word) and choose another data / field as the fourth dimension that will be displayed as a point with dimension / radius according to the magnitude of that data / field. It is easy to see through this visualization that there is a very big difference between "left" and "right" thread products, as well as between the various measures (represented in the $\mathrm{ZZ}$ axis).

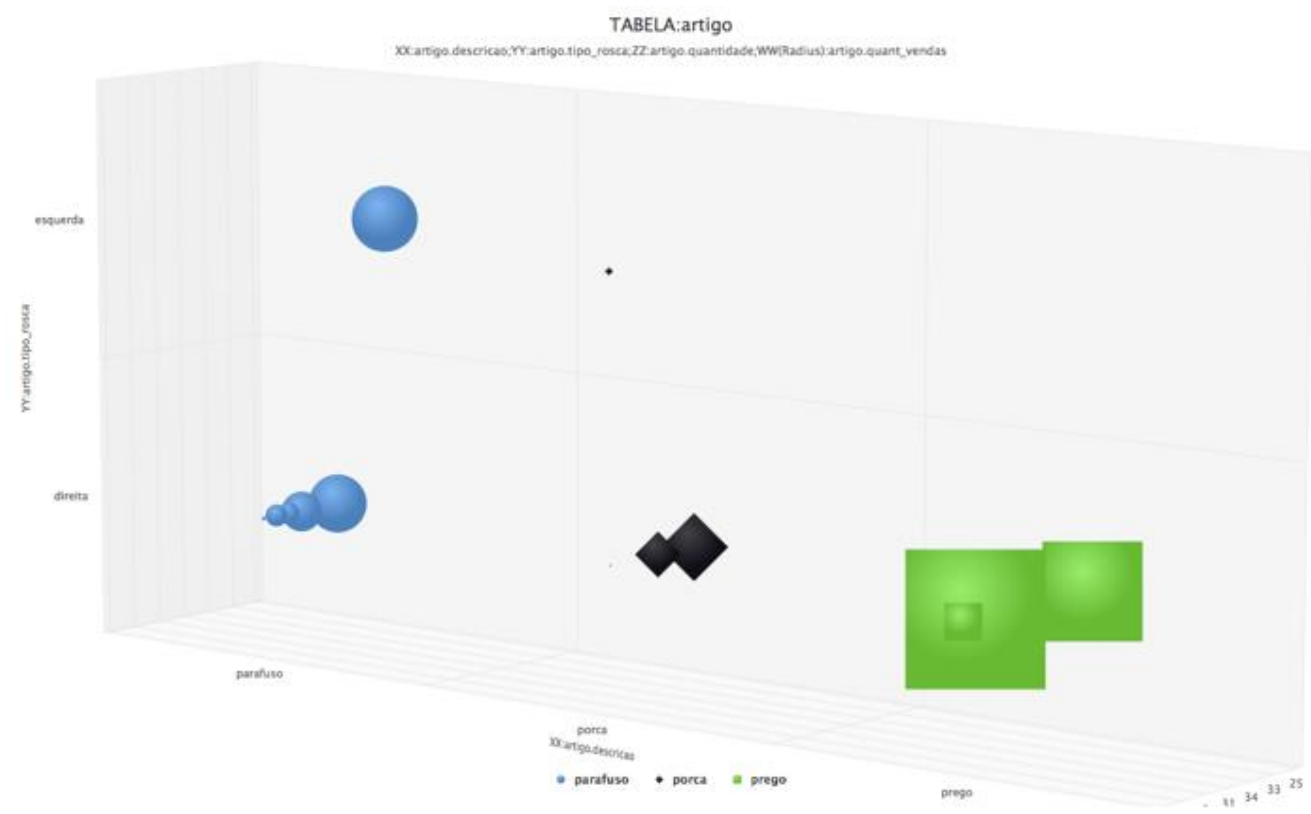

Figure 8. 3D+1 Data Mining "Radius Points"

At this stage of the implementation of this prototype, the objective was to allow the user, regardless of his competencies and privileges within the company, to view the data he selected most in a dynamic and easy way associated with meaningful visualizations.

\section{CONCLUSION AND FUTURE WORK}

It is important that a user can view what he wants, when he wants and how he wants. If these visualizations are associated, help for an easy interpretation of the data, even better. An incorrect choice of display type can prevent you from obtaining the desired information. The development of a software tool to support the design of the user interface of a DSS is not sufficient in itself and should, as far as possible, meet the specifics of each user. For example, while information may be displayed graphically, users may not be able to understand everything that is being displayed or they may be overwhelmed with too much data to be displayed on the monitor. A good system should help users to find meaningful information in a simple way, it should be friendly and customizable, and also suggest the best visualization to obtain that information.

Visualizing the information contained in the data by means of graphs or images is a technology in development that transforms traditional methods of observing and analyzing relational data tables so that people can discover relationships and structure in a more intuitive way. Thus, hidden information can be easily discovered. A good DSS visualization tool can be very important and advantageous in making decisions, supporting managers and leaders to make the right decision. As far as possible, DSS should be easy, simple, effective and customizable. Data visualization and, therefore, information play a fundamental role in decision making, supported by decision support systems, but we believe that the challenges are still in the beginning.

With the MLV-Viewer, it was intended to implement a dynamic and interactive system, in which the user can choose the best way to view the data. Converting simple data into meaningful information through data mining. MLV-Viewer has the following advantages: multiplatform; free; adaptable to the user; in real time; a system that allows importing and exporting data, various types of visualizations and with multiple dimensions, making analysis and forecasting of data; universal. As disadvantages can be pointed out: lacking the necessary tests, both of robustness and usability, among others; allow more user configurations. 
As the future work it is intended that this prototype advises the user on a set of views that are more recommended or recommended for the industry in question. On the other hand, it is intended to implement more methods of data mining and to develop methods of extrapolation and data prediction.

\section{REFERENCES}

Ashraf, S., \& Khan, S. A. (2015). Visualizations-based analysis of Telco data for business intelligence. Proceedings of the IEEE International Conference on Software Engineering and Service Sciences, ICSESS, 2015-Novem, $242-246$. https://doi.org/10.1109/ICSESS.2015.7339046

Cota, Manuel Pérez; Castro, M. R. G., \& Domínguez, J. Á. (2014). Importance of visualization usage in enterprise decision making environements. In 2014 9th Iberian Conference on Information Systems and Technologies (CISTI) (Ed.), Importance of visualization usage in enterprise decision making environements. Retrieved from 20149 th Iberian Conference on Information Systems and Technologies (CISTI)

Ellouzi, H. ;, Ltifi, H. ; \& Ayed, M. Ben. (2016). New Multi-Agent architecture of visual Intelligent Decision Support Systems application in the medical field. IEEE/ACS International Conference on Computer Systems and Applications, AICCSA (2016) 2016-July. https://doi.org/10.1109/AICCSA.2015.7507135

Grignard, A., Drogoul, A., \& Zucker, J. (2013). A Model-View / Controller approach to support visualization and online data analysis of Agent-Based Simulations. 233-236. 2013 IEEE RIVF International Conference on Computing \& Communication Technologies - Research, Innovation, and Vision for the Future (RIVF).

Groumpos, P. P. (2016). Conceptual Modeling and Decision Making Support Systems for Complex Dynamical Systems : A Critical Overview (IEEE, ed.). IEEE.

Huru, D., Leordeanu, C., Apostol, E., \& Cristea, V. (2018). BigClue: Towards a generic IoT cross-domain data processing platform. Proceedings - 2018 IEEE 14th International Conference on Intelligent Computer Communication and Processing, ICCP 2018, 427-434. https://doi.org/10.1109/ICCP.2018.8516597

Jorgensen, M., Spohn, J., Bunn, C., Dong, S., Li, X., \& Kaeli, D. (2018). An interactive big data processing/visualization framework. In U. 20172017 IEEE MIT Undergraduate Research Technology Conference (Ed.), 2017 IEEE MIT Undergraduate Research Technology Conference, URTC 2017 (Vol. 2018-Jan, pp. 1-4). https://doi.org/10.1109/URTC.2017.8284188

Kumar, S. M., \& Belwal, M. (2018). Performance dashboard: Cutting-edge business intelligence and data visualization. Proceedings of the 2017 International Conference On Smart Technology for Smart Nation, SmartTechCon 2017, 1201-1207. https://doi.org/10.1109/SmartTechCon.2017.8358558

Müller, H., Reihs, R., Posch, A. E., Kremer, A., Ulrich, D., \& Zatloukal, K. (2016). Data Driven GUI Design and Visualization for a NGS based Clinical Decision Support System. 2-7. https://doi.org/10.1109/IV.2016.79

Wu, X., Kumar, V., Ross, Q. J., Ghosh, J., Yang, Q., Motoda, H., ... Steinberg, D. (2008). Top 10 algorithms in data mining. Knowledge and Information Systems, 14(1), 1-37. https://doi.org/10.1007/s10115-007-0114-2

Yan, X., Qiao, M., Li, J., Simpson, T. W., Stump, G. M., \& Zhang, X. (2012). A work-centered visual analytics model to support engineering design with interactive visualization and data-mining. Proceedings of the Annual Hawaii International Conference on System Sciences, 1845-1854. https://doi.org/10.1109/HICSS.2012.87 